\title{
Activation of Alpha7 Nicotinic Acetylcholine Receptors Reduce Chronic Pain-Induced Depression-Like Behaviors Via WNT/ $\beta$-Catenin Pathway
}

Yongmei Chen ( $\square$ chenyongmeicym@sina.com )

Institution of Neurosciences

Xingrui Gong

department of neuroscience

Wen Wen

institution of neuroscinece

Meihua Cai

Institute of neuroscience

Mazhong Zhang

institute of neuroscience

\section{Research}

Keywords: alpha7 nicotinic acetylcholine receptor, pain, depression, WNT/ $\beta$-catenin, inflammation

Posted Date: May 7th, 2021

DOI: https://doi.org/10.21203/rs.3.rs-488235/v1

License: (c) (i) This work is licensed under a Creative Commons Attribution 4.0 International License.

Read Full License 


\section{Abstract}

Chronic pain frequently leads to depression and microglia-related inflammation is the common mechanism for chronic pain and depression. Activation of alpha 7 nicotinic acetylcholine ( $\mathrm{a} 7 \mathrm{nAch}$ ) receptor suppresses microglia-related inflammation. Thus, we evaluated the activation of a7 nAch receptors on chronic pain-induced depression-like behaviors and explored the potential mechanisms.

Methods: Male Wistar rats that received spared nerve injury (SNI) and complete Freund's adjuvant (CFA) into the unilateral ankle articular cavity received Open field, sucrose preference, and pain behavior tests. The effect of intracerebroventricular (ICV) injection of a7 nAch receptor agonist PHA and a7 nAch receptor antagonist on depression-like behaviors and cytokines expression was examined. Cytokines were measured in the brain using Enzyme-linked immune absorbent assay.

Results: SNI surgery and CFA injection induced depression-like behaviors, and ICV injection of PHA reduced that depression-like behaviors. SNI surgery and CFA injection skewed the medial prefrontal cortex, basolateral amygdala, and ventral hippocampus to a pro-inflammatory response and ICV injection of PHA shift brain from pro-inflammatory to anti-inflammatory response in that sites. The anti-depressive effect and anti-inflammatory effect of PHA were reversed by WNT/ $\beta$-catenin inhibitor.

Conclusions: Activation of alpha7 nicotinic acetylcholine receptors reduce chronic pain-induced depression-like behaviors, the mechanisms may be attributed to the activation of WNT/ $\beta$-catenin pathway and suppression of brain inflammation.

\section{Background}

Chronic pain is detrimental to human beings and frequently causes psychiatric disorders such as depression [1]. Although effective treatments are available, about one third of patients with depression fail to respond to conventional antidepressant therapies [2], which lead to searching new therapeutic approaches. Recently, depression has been regarded as an inflammatory disease, because suppression of inflammation improved the depressive behaviors. The proinflammatory cytokines affect neuronal activity and results in depression-like behaviors. The inflammation mainly comes from microglia, as minocycline, a microglial inhibitor suppresses brain inflammation and alleviates the depressive behaviors[3]. Thus, targeting microglial-related inflammation is an effective approach to managing psychiatric illness.

Alpha 7 nicotinic acetylcholine ( $\mathrm{a} 7 \mathrm{nAch}$ ) receptor, a subtype of nicotinic acetylcholine receptors, is involved in a variety of biological functions such as synaptic plasticity and neuronal survival; and is considered a prominent therapeutic target in neuropathological diseases[4]. Microglia, an immune cell in the central nervous system, express a7 nAch receptors, and by targeting a7 nAch receptor could change the microglia status and resolve microglial-related neuroinflammation[5]. In recent years, "cholinergic antiinflammatory pathway" has been demonstrated to regulate central nervous inflammation via the a7 nAch receptor of microglia[6]. Activation of microglia a7 nAch receptor also protect the brain from various 
insults by decreasing the proinflammatory effect of microglia[7]. Thus, activation of a7 nAch receptor may have anti-psychiatric effect.

PHA-543613 (PHA) is an a7 nAch receptor agonist, and we have previously shown that PHA prevent the development and maintenance of neuropathic pain by suppression of microglia-related inflammation[8]. Since chronic pain frequently results in depression and they share a common biological change of inflammation in the central nervous [9], whether targeting a7 nAch receptors can be used for treating chronic pain-induced depression remain to be clarified. We design this experiment to test the effect of ICV (intracerebroventricular) injection of a7 nAch receptor agonist PHA on the depression-like behaviors induced by SNI surgery and CFA injection and explore the potential signal pathways involved in the antidepressive effect of $a 7$ nAch receptor activation.

\section{Methods}

\section{Animals and drugs preparation}

Our experiments were performed in accordance with the Animal Care and use Committee of Shanghai Jiao Tong University and animal care guidelines of the National Institutes of Health. Male Wistar rats were obtained from the Shanghai Experimental Animal Institute and were kept in a temperature and humidity-controlled environment on a 12-h light/dark cycle (light on between 8:00 AM. to 18:00 P.M.) with ad libitum access to food and water. The a7 nAchR agonist, PHA, and its antagonist, methyllycaconitine citrate (MLA) were purchased from Tocris Biosciences (Minneapolis, USA). PHA was dissolved in 5\% dimethylsulfoxide (DMSO) at a dosage of $4 \mu \mathrm{g}$ in a volume of $4 \mu \mathrm{L}$. MLA was dissolved in normal saline and prepared at a dosage of $4 \mu \mathrm{g}$ in a volume of $4 \mu \mathrm{L}$. For the control group, an equal volume of $5 \%$ DMSO was used according to our previous study. Minocycline hydrochloride (purchased from SigmaAldrich Corp, St Louis, USA) was dissolved in normal saline with a concentration of $40 \mu \mathrm{g} / \mu \mathrm{L}$. SB431542 (a TGF $\beta$ inhibitor; Sigma-Aldrich, St. Louis, USA) were dissolved in $4 \%$ DMSO $(4 \mu \mathrm{l}, 5 \mathrm{nmol})$ and used for ICV injection[10]. AZD8055(a mTOR inhibitor; Sigma-Aldrich, St. Louis, USA) was prepared $10 \mathrm{mg} / \mathrm{mL}$ with $4 \%$ DMSO as vehicle and used $4 \mu \mathrm{L}$ for ICV injection[11]. IWR-1-endo[12] (IWR, a WNT/ $\beta$-catenin inhibitor), Numb[13] (a notch inhibitor) and AZD1480[14] (a JAK inhibitor) were bought from SigmaAldrich, St. Louis, USA and were dissolved in $4 \%$ DMSO with a concentration of 5 uM and a volume of 4 $\mu \mathrm{L}$ was used for ICV injection.

\section{Animal models establishments}

Spared nerve injury (SNI) surgery was performed according to our previous study[15] . Briefly, rats received general anesthesia with isoflurane $5 \%$ induction and $2.5 \%$ maintenance using an inhalational anesthesia system (Penlon Sigma Delta, Louisville, KY, USA). The rats were fixed on an operation table and the sciatic nerve was exposed at the lateral thigh with an animal surgery microscope (Leica M205FA, Germany); the tibia and common peroneal branches were dissected and tightly ligated with 6-0 absorbable suture and transected, leaving the sural nerve intact. The sciatic nerve of the sham group was 
only exposed, without ligation and transection. Then the skin was sutured with 5-0 sutures. The rat was returned to their cages for recovery as the surgery was over.

Monoarthritic model was induced by an injection of complete Freund's adjuvant (CFA) into the unilateral ankle articular cavity[16]. The experimental rats were anesthetized with isoflurane prior to inducing arthritis. Then the skin around the site of injection was sterilized with $75 \%$ alcohol and the right leg of the rat was held, and the fossa of the lateral malleolus of the fibula was located. A 28-gauge needle was used vertically to penetrate the skin, and turned distally to insert into the articular cavity from the gap between the tibiofibular and tarsus bone until a distinct loss of resistance. Then, a volume of $50 \mathrm{ul}$ of CFA was injected. For the sham group, the same amount of normal saline was injected into the right ankle articular cavity.

For the food intake evaluation, $50 \mathrm{~g}$ food was provided for each rat in one cage and determined at the indicated day for $24 \mathrm{hs}$. The amount of food remaining after $24 \mathrm{hs}$ was individually weighed and subtracted from the original quantity provided. Body weight was recorded before surgery or stimulation, before and after medical intervention. All rats were free access to water.

\section{Pain behavior tests}

For mechanical allodynia, the values were measured according to our previous study[17]. Briefly, the rats were placed in a Plexiglas box on a mesh floor $50 \mathrm{~cm}$ above the table. After $15 \mathrm{~min}$ of acclimation, the mechanical withdrawal threshold was measured with an electronic apparatus (IITC Life Science, Woodland Hills, CA, USA). An increasing force was used to stimulate the lateral side of the ipsilateral hind paw. As an immediate paw withdrawal was observed, the value was recorded; the mean value was averaged from 3 repeated measures with an interval of 5 min.

Response rate to cold acetone was done according to our previous study[17]. The positive response was recorded as withdrawal or licking or shaking of the hind paw to a drop of acetone applied to the ventral surface of the paw. The acetone response was repeated for 5 times and the response rate was calculated.

\section{Depression-like behavior tests}

The open field test was done according to our previous study[17]. The rat was placed in a square arena $(50 \times 50 \times 50 \mathrm{~cm})$, which was divided into two areas: a peripheral area and a central $(17 \times 17 \mathrm{~cm})$ square area. The center distance and total distance were recorded in the experiment, and center/total distance duration were calculated as measures of depression-like behavior, and total running distance was recorded as a measure of general motor behavior. The running track was recorded for $5 \mathrm{~min}$ by a camera $2 \mathrm{~m}$ above the box. The field was cleaned with $75 \%$ ethanol after each test.

The sucrose preference experiment followed a previous study with minor modifications[17]. Briefly, all of the rats were habituated with $1 \%$ sucrose for $48 \mathrm{~h}$ before the experiments. On the day of the experiment, each rat received an 8-h fluid and food deprivation and then was exposed to two bottles for $1 \mathrm{~h}$, one filled 
with $1 \%$ sucrose and the other tap water. The sucrose intake percentage was calculated with a formula: (sucrose/(sucrose + water)) $\times 100 \%$.

\section{Intracerebroventricular catheter placement and injection}

Intracerebroventricular (ICV) catheter placement and injection followed the procedures used in our previous study[17]. Briefly, rats were anesthetized with intraperitoneal injection of pentobarbital sodium $(50 \mathrm{mg} / \mathrm{kg}$ ). Then, the rat was fixed on a stereotaxic apparatus. The surgery was done and a catheter was placed with stereotaxic coordinates: $0.8 \mathrm{~mm}$ posterior, $1.5 \mathrm{~mm}$ left lateral, and $4.5 \mathrm{~mm}$ ventral from the bregma. The guide cannula was fixed with dental cement and the rat was returned to individual cage for recovery. The ICV cannulation was verified by injection of methylene blue through the cannula after experiment. For each daily injection, rats were anesthetized with isoflurane as above; rats received bilateral microinjection of PHA and inhibitors or minocycline $(160 \mu \mathrm{g})$ or saline (control) into the cerebral ventricle. A total volume of $4.0 \mu \mathrm{l}$ was infused into each side over $10 \mathrm{~min}$, and the injection syringe was left in place for an additional $5 \mathrm{~min}$ to allow for diffusion. The microinjections were performed daily for six days, followed by behavior tests.

\section{Inflammatory cytokines measurements with enzyme-linked immune-absorbent assay}

After the last behavior tests, rats were deeply anesthetized with pentobarbital sodium $(100 \mathrm{mg} / \mathrm{kg})$ followed by decapitation according to our previous study[17]. Tissues were collected for ELISA followed our previously study. Briefly, the brains were placed in a chilled matrix and micro-dissected on a chilled glass plate. The medial prefrontal cortex (mPFC) was obtained from a 2-mm-thick slice ranging from approximately 5 to $3 \mathrm{~mm}$ anterior of Bregma. The ventral hippocampus (VH) was isolated from a 4-mmthick section ranging from approximately 3.2 to $7.2 \mathrm{~mm}$ posterior of Bregma and was separated from cortex and underlying brain structures. The basolateral amygdala (BA) was dissected out ranging from approximately 1.2 to $3.2 \mathrm{~mm}$ posterior of Bregma just lateral to the optic tracks. After the samples were collected, these tissues were homogenized with normal saline and centrifuged at $4000 \mathrm{rpm}, 4^{\circ} \mathrm{C}$ for 10 min. The supernatants were used for IL-10, IL-1 $\beta$, TNF- $\alpha$ and IL-18 assay using ELISA kits (Nanjingjiancheng Biocompany, Nanjing, China) and the procedures followed the manufacturer's instructions

\section{Statistical analysis}

The data are shown as mean \pm SD and were analyzed with one-way or two-way analysis of variance (ANOVA) with repeated measures. Bonferroni post hoc analysis was conducted if a statistically significant difference was found. Nonparametric Wilcoxon or unpaired " $t$ " test was performed for comparison of non-repeated measures. $P<0.05$ was considered statistically significant. The statistical analysis was performed using GraphPad Prism software (GraphPad Prism 5.0, GraphPad Software Inc., San Diego, CA, USA).

\section{Results}




\section{SNI surgery induced depression-like behaviors after surgery}

The depression-like behaviors were measured from POD14 onward, and the results showed that SNI surgery decreased center distance and center/total distance compared to sham group in the open field test from POD7 to POD 21 after surgery (Fig $1 \mathrm{~A}$ and $\mathrm{C}$ respectively). SNI surgery did not affect total running distance in the open field test after surgery (Fig 1B). In the sucrose preference test, SNI decreased sucrose intake percentage from POD7 to POD21(Fig 1D). In addition, SNI decreased food intake and weight gain compared to sham after surgery from POD7 to POD21(Fig $1 \mathrm{E}$ and $\mathrm{F}$ respectively). The paw withdrawal threshold and response to acetone were recorded before and after SNI surgery. The results showed that SNI surgery decreased paw withdrawal threshold and increased response to acetone after surgery from POD7 to POD21(Fig $1 \mathrm{G}$ and $\mathrm{H}$ respectively).

\section{ICV injection of a7 nAch receptor agonist PHA and microglia inhibitor MIN decreased depression-like behaviors after SNI surgery}

The behavior results showed that SNI surgery decreased center distance and center/total distance from POD10 to POD18, and ICV PHA or MIN improved that decrement of center distance and center/total distance on POD18 (Fig 2A and C respectively). SNI decreased sucrose intake percentage from POD10 to POD18 and ICV PHA or MIN decreased that effect on POD18 (Fig 2D). SNI surgery decreased food intake and weight gain from POD10 to POD18 compared to sham group, and ICV PHA or MIN decreased that effect on POD18 (Fig 2E and F respectively). Although SNI decreased paw withdrawal threshold and increased response to acetone, ICV PHA or MIN did not affect paw withdrawal threshold and response to acetone (Fig $2 \mathrm{G}$ and $\mathrm{H}$ respectively). The time course for surgery and experiment was shown in Fig $2 \mathrm{I}$.

\section{PHA decreased depression-like behaviors after SNI surgery by the activation of a7 nAch receptors and suppression of brain inflammation}

The results showed that ICV co-administration of a7 nAch receptor antagonist MLA decreased antidepressive effect of PHA in center distance and center/total distance in the open field test (Fig $3 \mathrm{~A}$ and $\mathrm{C}$ respectively) and the total distance running was not different between groups after SNI surgery on POD18 (Fig 3B). PHA increased sucrose intake percentage and food intake and weight gain after SNI, while coadministration MLA decreased that effect (Fig 3D, E and F respectively). Both MLA did not affect the effect of PHA on paw withdrawal threshold and response to acetone (Fig $3 \mathrm{G}$ and $\mathrm{H}$ respectively).

In addition, ICV PHA increased IL-10 in the MPFC, BA and VH compared to SNI+Vehicle group, and MLA decreased that effect (Fig 4A, E and I respectively). However, SNI increased IL-1 $\beta$ in the MPFC, BA and VH compared to Naive group, and ICV PHA decreased IL-1 $\beta$ in the MPFC, BA and VH compared to $\mathrm{SNI}+$ Vehicle group, while MLA decreased the effect of PHA (Fig 4B, F and J respectively). Similarly, SNI increased TNF- $\alpha$ (Fig 3C, G and $K$ respectively) and IL-18 (Fig 4D, H and L respectively) in the mPFC, BA and $\mathrm{VH}$ compared to Naive group, and ICV PHA decreased their content in the MPFC, BA and VH compared to SNI+Vehicle group, while MLA decreased the effects of PHA. 
PHA decreased depressive-like behaviors after CFA injection by the activation of a7 nAch receptors and suppression of brain inflammation

In the CFA experiment, the CFA injection and ICV cannulation were performed on the same day after anesthesia. The results showed that CFA decreased center distance and center/total distance in the open field test, and did affect total running distance moved, compared to naïve group, and PHA improved the behaviors in the open filed tests, whereas MLA decreased the effect of PHA (Fig 5A, B and C respectively). CFA decreased sucrose intake percentage, food intake and weight gain, and PHA reversed that effect, and the effects was abrogated by MLA (Fig 5D, E and I respectively). CFA decreased mechanical anodynia and increased response to cold, whereas ICV PHA and MLA did not affect that response (Fig 5G and H respectively). The research protocol was shown in Fig 51 .

CFA did not affect IL-10 content in the MPFC BA and VH, and ICV PHA increased IL-10 content in the mPFC and BA, and the effect was decreased by MLA (Fig 6A). CFA increased IL-1 $\beta$ content in the mPFC $B A$ and $V H$, and ICV PHA decreased IL-1 $\beta$ increment in that sites, and the effect was decreased by MLA (Fig 6B). CFA increased TNF-a content in the MPFC BA and VH, and ICV PHA decreased TNF-a content in the MPFC, BA and VH, the effect was abolished by MLA (Fig 6C). CFA increased IL-18 content in the mPFC BA and VH, and ICV PHA decreased IL-18 content in the MPFC and VH, while the effect of PHA was decreased by MLA (Fig 6D).

\section{PHA decreased depressive-like behaviors via WNT/ $\beta$-catenin signal pathway}

PHA increased center distance, center/total distance ratio, sucrose intake percentage, food intake and weight gain after SNI surgery and signal pathway inhibitors of TGF $\beta$, mTOR, WNT/ $\beta$, notch, JAK were coadministered to explore the potential anti-depressive mechanism of PHA. The results found WNT/ $\beta$ catenin inhibitor IWR reversed the anti-depressive effect of PHA on center distance, center/total distance ratio, sucrose intake percentage, food intake percentage and weight gain (Fig 7A, B, C, D, E and F respectively). Surprisingly, mTOR inhibitor AZD8055 also reversed the effect of PHA on food intake percentage and weight gain (Fig 7E and F respectively). And TGF $\beta$, notch and JAK pathway inhibitors did not affect anti-depressive behaviors of PHA.

\section{PHA decreased brain inflammation via WNT/ $\beta$-catenin and mTOR pathway}

The cytokines were measured after behavior tests. The results showed that PHA increased IL-10 and decreased IL-18 in mPFC, BA and VH induced after SNI surgery, and WNT/ $\beta$-catenin inhibitor IWR reversed those effect (Fig 8A and D respectively). In addition, PHA decreased IL-1 $\beta$ content in $\mathrm{mPFC}$ and VH after SNI surgery, however, the change was reversed by mTOR inhibitor AZD8055 and WNT/ $\beta$-catenin inhibitor IWR (Fig 8B). Similarly, PHA decreased the content of TNF in mPFC, BA and VH after SNI surgery, and the content change of TNFain mPFC and BA was reversed by mTOR inhibitor AZD8055 and WNT/ $\beta$-catenin inhibitor IWR (Fig 8C).

\section{Discussion}


Our results showed that ICV a7 nAch receptor agonists PHA decreased depression-like behaviors induced by $\mathrm{SNI}$ and CFA, the mechanisms could be due to the activation of WNT/ $\beta$-catenin signal pathway and suppression of brain inflammation.

Pain and depression comorbidity have been observed in clinics and investigated in animal models[18]. In the study, we found that SNI-induced depression-like behaviors after surgery. The results were confirmed by decreased center and center/total distance in the open field tests, and decreased sucrose and food intake, and weight gain compared to naïve rats. SNI surgery did not affect the total distance moved in the open field test, which excluded the possibility that the effect was attributed to motor impairment. Pain behaviors of paw withdrawal threshold and response to acetone suggest the successful establishment of pain animal models. In addition, animals received CFA injection, which is proinflammatory and induces pain, also present depressive behaviors, which confirm chronic pain induces depressive conditions. Clinical study has demonstrated that chronic pain frequently results in depression[1], and chronic pain contributed to the recurrence, longer duration and increased sadness of depressive episodes[19]. In addition, pain and depression were intertwined and co-exacerbate physical and psychological symptoms. The mechanism may be explained by some overlap biological process[20]. For example, injuries to sensory pathways have been demonstrated to affect the same brain regions that are involved in mood disorders[9].

Recently, depression has been shown to be an inflammatory disease, and inhibition of inflammation reduces depression-like behaviors[3]. In clinical study, neuroinflammation of cytokines has been analyzed in CSF of post-mortem brain tissue, and results found immune cells are involved in the immune response using both postmortem tissue and positron emission tomography (PET) imaging[21].The specialized immune cells in the brain are called microglia that comprise $5 \%-10 \%$ of total brain cells and their main functions are to maintain CNS homeostasis and to cope with damage or infection[22]. In many neurodegenerative and neuropsychiatric diseases, microglia are activated and contribute to pathology by promoting neuroinflammation[23]. In depressive conditions, microglia are activated and induce cascade proinflammatory response in the brain and contribute to the neuropathology and depression-like behaviors[24]. Evidence in rodents found that after insult, microglial indoleamine 2, 3-dioxygenase and kynurenine pathway are activated and generate excessive neurotoxic quinolinic acid, microglia shift to proinflammatory profile, and generates a host of cytokines, including interleukin IL-1 $\beta$, TNF- $\alpha$ and so on[25]. The proinflammatory quinolinic acid and cytokines from microglia inhibit hippocampal neurogenesis and induce depression-like behaviors, whereas treatment with minocycline, a microglia inhibitor, counteracted this effect. Thus, suppression of the brain microglia-related inflammation is effective approach to treating depression.

PHA is a a7 nAch receptor agonist, and our results showed that ICV injection of PHA decreased depression-like behaviors, including open field test, sucrose preference test and food intake induced by $\mathrm{SNI}$, the reason could be attributed to the activation of $\mathrm{a} 7 \mathrm{nAch}$ receptors, as co-administration of $\mathrm{a} 7$ nAch receptor antagonist MLA abolished that effect. Consistently, our previous study showed that IT injection of PHA prevents the development and maintenance of chronic pain. The reason is attributed to 
suppression of proinflammatory response in the spinal cord. In addition, PHA decreased inflammatory cytokines release from microglia in vitro by activation of $\mathrm{a} 7 \mathrm{nAch}$ receptors in primary microglia cultures[8]. Study have demonstrated that the inflammation in the brain majorly comes from microglia, as microglia suppression prevents the depression-like behaviors in animals[3]. In our study, we included a group of rats received MIN, a microglia inhibitor, and results showed that ICV MIN decreased SNI-induced depression-like behaviors and brain proinflammatory response. This confirmed depression is a microglial inflammatory disease and suppression of microglia-related inflammation reversed depression-like behaviors induced by SNI, which is consistent with our previous study.

The cholinergic anti-inflammatory pathway controls cytokine production and inflammation in the body[26]. Experimental evidence suggests that cholinergic anti-inflammatory signaling requires a7 nAch receptors expressed on non-neuronal cytokine-producing microglia in the central nervous system. a7 nAch receptor agonists inhibit cytokine release and shows protective effect in a variety of experimental lethal inflammatory models and has been exploited to counteract abnormal chronic and hyper-activated pro-inflammatory conditions[6]. Our study confirmed that suppression of inflammation in the brain is a promising therapeutic target for treating depression by activation of a7 nAch receptors. In order to confirm that the effect of a7 nAch receptor activation is not limited to SNl-induced depression, we included CFA model to prove the general effect of $a 7$ nAch receptor agonist in chronic pain-induced depression. Our results showed that CFA-induced depression-like behaviors, including decreased center distance, center/total distance, sucrose intake percentage and food intake, and ICV PHA provided antidepressive effect induced by CFA. In addition, the anti-depressive effect of PHA was reversed by MLA, which confirmed that PHA's anti-depressive effect by activation of $a 7$ nAch receptors. Further, PHA decreased CFA-induced brain inflammation, including increased IL-10, decreased IL-1 $\beta$, TNF- $\alpha$ and IL-18 in the brain.

To further explore the mechanisms of the anti-depressive effect of PHA, signal pathway inhibitors were administered at the same time. The results showed that WNT/ $\beta$-catenin inhibitor IWR reversed the antidepressive effect of PHA in the open field test and sucrose preference test, and both WNT/ $\beta$-catenin inhibitor IWR and mTOR inhibitor suppressed food intake and weight gain, whereas TGF- $\beta$, Notch, and JAK pathway inhibitors did not affect that effect. This suggests the anti-depressive effect of PHA through WNT/ $\beta$-catenin, while feeding behavior of food and weight gain are affected by both WNT/ $\beta$-catenin and mTOR signal pathway. WNT/ $\beta$-catenin are secreted glycoproteins that signals through the frizzled receptors and are essential for normal embryonic development $[27,28]$. Study have demonstrated WNT/ $\beta$ catenin signaling is associated in depression and antidepressant response. The WNT/ $\beta$-catenin pathway in nucleus accumbens is down-regulated in the mouse model of social defeat model of depression, and knock down of the signaling renders mice more susceptible to social defeat stress and promotes depression-like behaviors[29]. In addition, different classes of antidepressant treatments, including SSRIs, SNRI, and chronic electroconvulsive shock, showed anti-depressive effect by activation of WNT/ $\beta$-catenin pathway[30]. However, our study suggests activation of a7 $\mathrm{nAch}$ receptors showed anti-depressive effect via the WNT/ $\beta$-catenin pathway. 
Our result showed that the mTOR inhibitor decreased weight gain of PHA. the reason may be attributed to that mTOR controls the body metabolism and increases food intake and consequently result in weight gain[31]. Thus, food intake and weight gain are also affected by mTOR inhibitors in our experiments. The inflammatory cytokine concentration was measured and the results showed WNT/ $\beta$-catenin inhibitor IWR reversed the IL-10, IL-1 $\beta$, TNF- $\alpha$ and IL-18 content change induced by PHA, which confirmed PHA present its anti-inflammation and anti-depressive effect via WNT/ $\beta$-catenin signal pathway. Surprisingly, IL-1 $\beta$, TNF-a content change were reversed also by mTOR pathway inhibitors. This indicates that mTOR pathway is also anti-inflammatory in chronic pain-induced depressive animal models. Although previous study has demonstrated that the activation of mTOR reversed depression-like behaviors, the mechanisms may be explained by suppression of inflammation[32,33]. However, mTOR pathway inhibitor did not affect anti-depressive effect of PHA, and this suggested mTOR maybe a downstream signal of WNT/ $\beta$ catenin, or at least not a major contributor of the anti-depressive effect of PHA[33]. PHA activates WNT/ $\beta$ catenin and results in the increment of IL-10 and the decrement of IL-1 $1 \beta$, TNF- $\alpha$, IL 18 release, and at the same time, activates mTOR which result in enhanced decrement of IL-1 $\beta$ and TNF- $\alpha$ release. As previous study has demonstrated IL-10 can resolve the proinflammatory response, the anti-depressive effect of PHA should be attributed to the activation of WNT/ $\beta$-catenin and increased IL-10 release[34].

In the study, several cytokines were chosen to evaluate the brain inflammatory response. Previous studies have demonstrated that IL-10, IL-1 $\beta$, TNF- $a$ and IL-18 were related to depression, and modulation of inflammation can induce or decrease depression-like behaviors. In our study, both SNI and CFA increased IL-1 $\beta$, TNF- $\alpha$ and IL-18 in the brain, and ICV PHA increased IL-10, and decreased IL-1 $\beta$, TNF- $\alpha$ and IL-18 content. This confirmed increased anti-inflammatory IL-10 suppressed pro-inflammatory response after chronic pain insult[35]. In addition, followed our previous study, tissue of MPFC BA and VH were acquired to testify the inflammatory profile after SNI or CFA, because those sites have been proved to be involved in depression and showed increased cytokines expression after insult[36]. Our research verified that these sites are proper and effective for the study of depression and anti-depressive treatment response.

\section{Conclusions}

Our study suggests that the activation of a7 nAch receptor presents anti-depressive-like behaviors, and the reason could be attributed to the activation of WNT/ $\beta$-catenin pathway and suppression of inflammation in the brain.

\section{Abbreviations}

a7 nAch

alpha 7 nicotinic acetylcholine; ANOVA:analysis of variance; BA:basolateral amygdala; CFA:complete Freund's adjuvant; DMSO:dimethylsulfoxide; ELISA:enzyme-linked immune-absorbent assay; ICV:intracerebroventricular; IWR:IWR-1-endo; MIN:minocycline; MLA:methyllycaconitine citrate; mPFC:medial prefrontal cortex; POD:postoperative day; PHA:PHA-543613; SNI:spared nerve injury; VH:ventral hippocampus; 


\section{Declarations}

Acknowledgement

The authors would like to thank all the person who participated in this study.

\section{Funding}

This research is funded by a grant from the National Natural Science Foundation of China (NSFC, Grant No. 81270414), Shanghai Municipal Commission of Health and Family Planning Founding for Key Developing Disciplines (No. 2015ZB0106) and Natural Science Foundation of Shanghai (20ZR1434600) and Natural Science Foundation of Hubei (2019CFB411).

\section{Authors' contributions}

Xingrui Gong and Yongmei Chen did the surgery and behavior tests and ELISA experiment. Meihau Cai and Wen Wen did the statistical analysis. Mazhong Zhang designed the experiment. All authors read and approved the final manuscript.

\section{Ethics approval and consent to participate}

All animal experiments were approved by the Experimental Animal Ethics Committee of Shanghai Jiao Tong University School of Medicine and Hubei University of Arts and Science, and were conducted under the guidelines of National Institute of Health.

\section{Availability of data and materials}

Available upon request.

\section{Consent for publication}

Not applicable.

\section{Competing interests}

The authors have no conflicts of interest to declare.

\section{References}

1. IsHak WW, Wen RY, Naghdechi L, Vanle B, Dang J, Knosp M, Dascal J, Marcia L, Gohar Y, Eskander L, et al. Pain and Depression: A Systematic Review. Harv Rev Psychiatry. 2018;26(6):352-63.

2. Rush AJ, Trivedi MH, Wisniewski SR, Nierenberg AA, Stewart JW, Warden D, Niederehe G, Thase ME, Lavori PW, Lebowitz BD, et al. Acute and longer-term outcomes in depressed outpatients requiring one or several treatment steps: a STAR*D report. Am J Psychiatry. 2006;163(11):1905-17. 
3. Yirmiya R, Rimmerman N, Reshef R. Depression as a Microglial Disease. TRENDS NEUROSCl. 2015;38(10):637-58.

4. Bencherif M, Lippiello PM, Lucas R, Marrero MB. Alpha7 nicotinic receptors as novel therapeutic targets for inflammation-based diseases. CELL MOL LIFE SCI. 2011;68(6):931-49.

5. Bagdas D, Gurun MS, Flood P, Papke RL, Damaj MI. New Insights on Neuronal Nicotinic Acetylcholine Receptors as Targets for Pain and Inflammation: A Focus on alpha7 nAChRs. CURR NEUROPHARMACOL. 2018;16(4):415-25.

6. Martelli D, McKinley MJ, McAllen RM. The cholinergic anti-inflammatory pathway: a critical review. Auton Neurosci. 2014;182:65-9.

7. Egea J, Buendia I, Parada E, Navarro E, Leon R, Lopez MG. Anti-inflammatory role of microglial alpha7 nAChRs and its role in neuroprotection. BIOCHEM PHARMACOL. 2015;97(4):463-72.

8. Ji L, Chen Y, Wei H, Feng H, Chang R, Yu D, Wang X, Gong X, Zhang M. Activation of alpha7 acetylcholine receptors reduces neuropathic pain by decreasing dynorphin $A$ release from microglia. BRAIN RES. 2019;1715:57-65.

9. Tappe-Theodor A, Kuner R. A common ground for pain and depression. NAT NEUROSCI. 2019;22(10):1612-4.

10. Caraci F, Battaglia G, Busceti C, Biagioni F, Mastroiacovo F, Bosco P, Drago F, Nicoletti F, Sortino MA, Copani A: TGF-beta 1 protects against Abeta-neurotoxicity via the phosphatidylinositol-3-kinase pathway. NEUROBIOL DIS 2008, 30(2):234-242.

11. Yang C, Ren Q, Qu Y, Zhang JC, Ma M, Dong C, Hashimoto K. Mechanistic Target of RapamycinIndependent Antidepressant Effects of (R)-Ketamine in a Social Defeat Stress Model. Biol Psychiatry. 2018;83(1):18-28.

12. Zhang YK, Huang ZJ, Liu S, Liu YP, Song AA, Song XJ. WNT signaling underlies the pathogenesis of neuropathic pain in rodents. J CLIN INVEST. 2013;123(5):2268-86.

13. Couturier L, Mazouni K, Schweisguth F. Inhibition of Notch recycling by Numb: relevance and mechanism(s). CELL CYCLE. 2013;12(11):1647-8.

14. Wei S, Luo C, Yu S, Gao J, Liu C, Wei Z, Zhang Z, Wei L, Yi B. Erythropoietin ameliorates early brain injury after subarachnoid haemorrhage by modulating microglia polarization via the EPOR/JAK2STAT3 pathway. EXP CELL RES. 2017;361(2):342-52.

15. Gong $X$, Chen $Y, F u$ B, Jiang J, Zhang M. Infant nerve injury induces delayed microglial polarization to the $\mathrm{M} 1$ phenotype, and exercise reduces delayed neuropathic pain by modulating microglial activity. NEUROSCIENCE. 2017;349:76-86.

16. Butler SH, Godefroy F, Besson JM, Weil-Fugazza J. A limited arthritic model for chronic pain studies in the rat. PAIN. 1992;48(1):73-81.

17. Gong X, Chen Y, Chang J, Huang Y, Cai M, Zhang M. Environmental enrichment reduces adolescent anxiety- and depression-like behaviors of rats subjected to infant nerve injury. J Neuroinflammation. 2018;15(1):262. 
18. Gerrits MM, van Oppen P, van Marwijk HW, Penninx BW, van der Horst HE. Pain and the onset of depressive and anxiety disorders. PAIN. 2014;155(1):53-9.

19. Ohayon MM. Specific characteristics of the pain/depression association in the general population. $J$ Clin Psychiatry. 2004;65(Suppl 12):5-9.

20. Meerwijk EL, Ford JM, Weiss SJ. Brain regions associated with psychological pain: implications for a neural network and its relationship to physical pain. BRAIN IMAGING BEHAV. 2013;7(1):1-14.

21. Williams M, Enna SJ. Prospects for neurodegenerative and psychiatric disorder drug discovery. Expert Opin Drug Discov. 2011;6(5):457-63.

22. Wolf SA, Boddeke HW, Kettenmann H. Microglia in Physiology and Disease. ANNU REV PHYSIOL. 2017;79:619-43.

23. Li Q, Barres BA. Microglia and macrophages in brain homeostasis and disease. NAT REV IMMUNOL. 2018;18(4):225-42.

24. Wohleb ES, Franklin T, Iwata M, Duman RS. Integrating neuroimmune systems in the neurobiology of depression. NAT REV NEUROSCI. 2016;17(8):497-511.

25. Garrison AM, Parrott JM, Tunon A, Delgado J, Redus L, O'Connor JC. Kynurenine pathway metabolic balance influences microglia activity: Targeting kynurenine monooxygenase to dampen neuroinflammation. PSYCHONEUROENDOCRINO. 2018;94:1-10.

26. Hoover DB. Cholinergic modulation of the immune system presents new approaches for treating inflammation. Pharmacol Ther. 2017;179:1-16.

27. Clevers H, Nusse R. Wnt/beta-catenin signaling and disease. CELL. 2012;149(6):1192-205.

28. Clevers H. Wnt/beta-catenin signaling in development and disease. CELL. 2006;127(3):469-80.

29. Wilkinson MB, Dias C, Magida J, Mazei-Robison M, Lobo M, Kennedy P, Dietz D, Covington HR, Russo $S$, Neve R, et al. A novel role of the WNT-dishevelled-GSK3beta signaling cascade in the mouse nucleus accumbens in a social defeat model of depression. J NEUROSCI. 2011;31(25):9084-92.

30. Okamoto H, Voleti B, Banasr M, Sarhan M, Duric V, Girgenti MJ, Dileone RJ, Newton SS, Duman RS. Wnt2 expression and signaling is increased by different classes of antidepressant treatments. Biol Psychiatry. 2010;68(6):521-7.

31. Deblon N, Bourgoin L, Veyrat-Durebex C, Peyrou M, Vinciguerra M, Caillon A, Maeder C, Fournier M, Montet $\mathrm{X}$, Rohner-Jeanrenaud $\mathrm{F}$, et al. Chronic $\mathrm{mTOR}$ inhibition by rapamycin induces muscle insulin resistance despite weight loss in rats. Br J Pharmacol. 2012;165(7):2325-40.

32. Abelaira HM, Reus GZ, Neotti MV, Quevedo J. The role of mTOR in depression and antidepressant responses. LIFE SCI. 2014;101(1-2):10-4.

33. Shimobayashi M, Hall MN. Making new contacts: the mTOR network in metabolism and signalling crosstalk. Nat Rev Mol Cell Biol. 2014;15(3):155-62.

34. Bluthe RM, Castanon N, Pousset F, Bristow A, Ball C, Lestage J, Michaud B, Kelley KW, Dantzer R. Central injection of IL-10 antagonizes the behavioural effects of lipopolysaccharide in rats. PSYCHONEUROENDOCRINO. 1999;24(3):301-11. 
35. Worthen RJ, Garzon ZS, Torres JC, Beurel E. Anti-inflammatory IL-10 administration rescues depression-associated learning and memory deficits in mice. J Neuroinflammation. 2020;17(1):246.

36. Belleau EL, Treadway MT, Pizzagalli DA. The Impact of Stress and Major Depressive Disorder on Hippocampal and Medial Prefrontal Cortex Morphology. Biol Psychiatry. 2019;85(6):443-53.

\section{Figures}
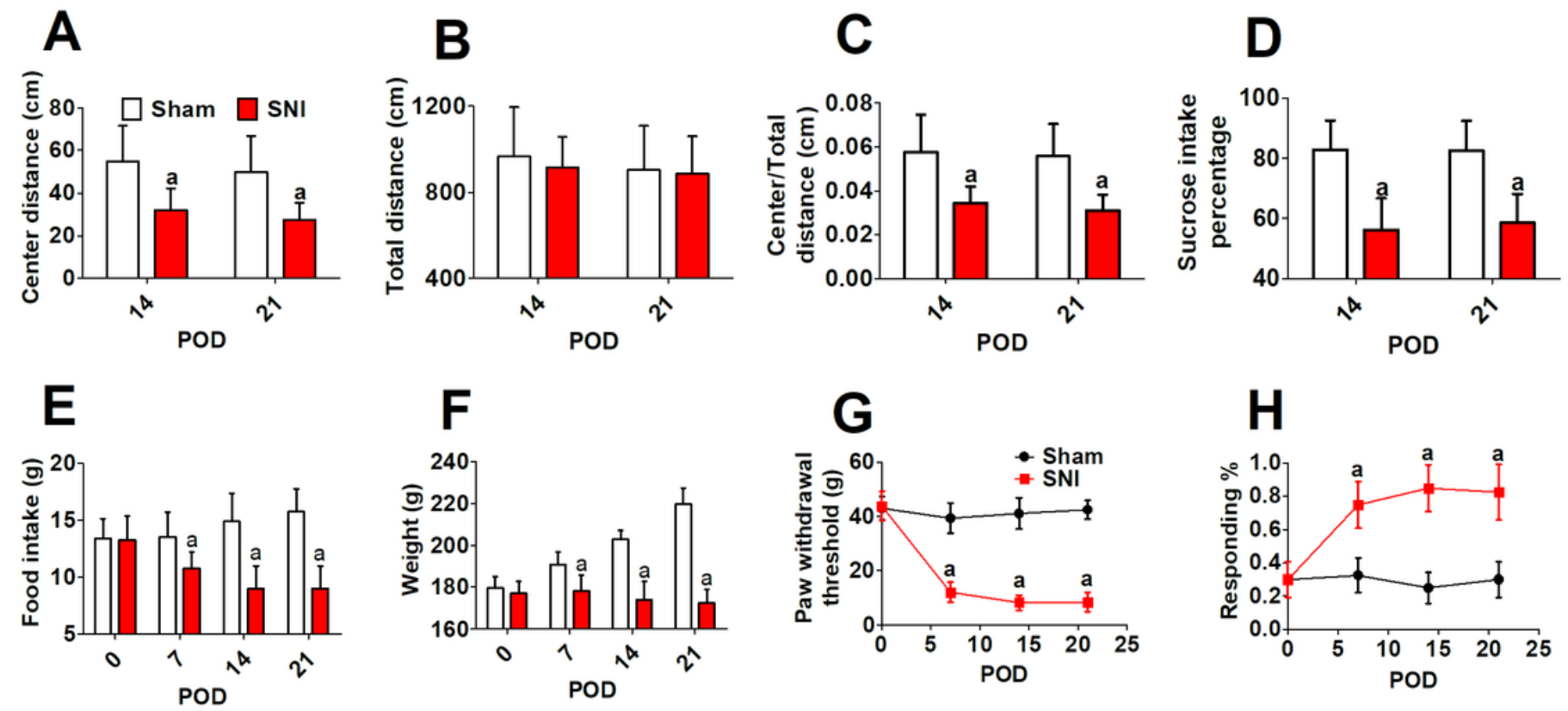

\section{Figure 1}

SNI surgery induced depression-like behaviors. SNI surgery decreased center distance (A) and center/total distance (C) compared to sham group in the open field test from POD7 to POD 21 after surgery. (B) SNI surgery did not affect total running distance in the open field test after surgery. (D) SNI decreased sucrose intake percentage from POD7 to POD21. In addition, SNI decreased food intake (E) and weight gain (F) compared to sham after surgery from POD7 to POD21. SNI surgery decreased paw withdrawal threshold $(G)$ and increased response to acetone $(H)$ after surgery from POD7 to POD21. $n=8$. "a" denotes the statistically significant difference compared to the sham group. SNI spared nerve injury, POD postoperative days 

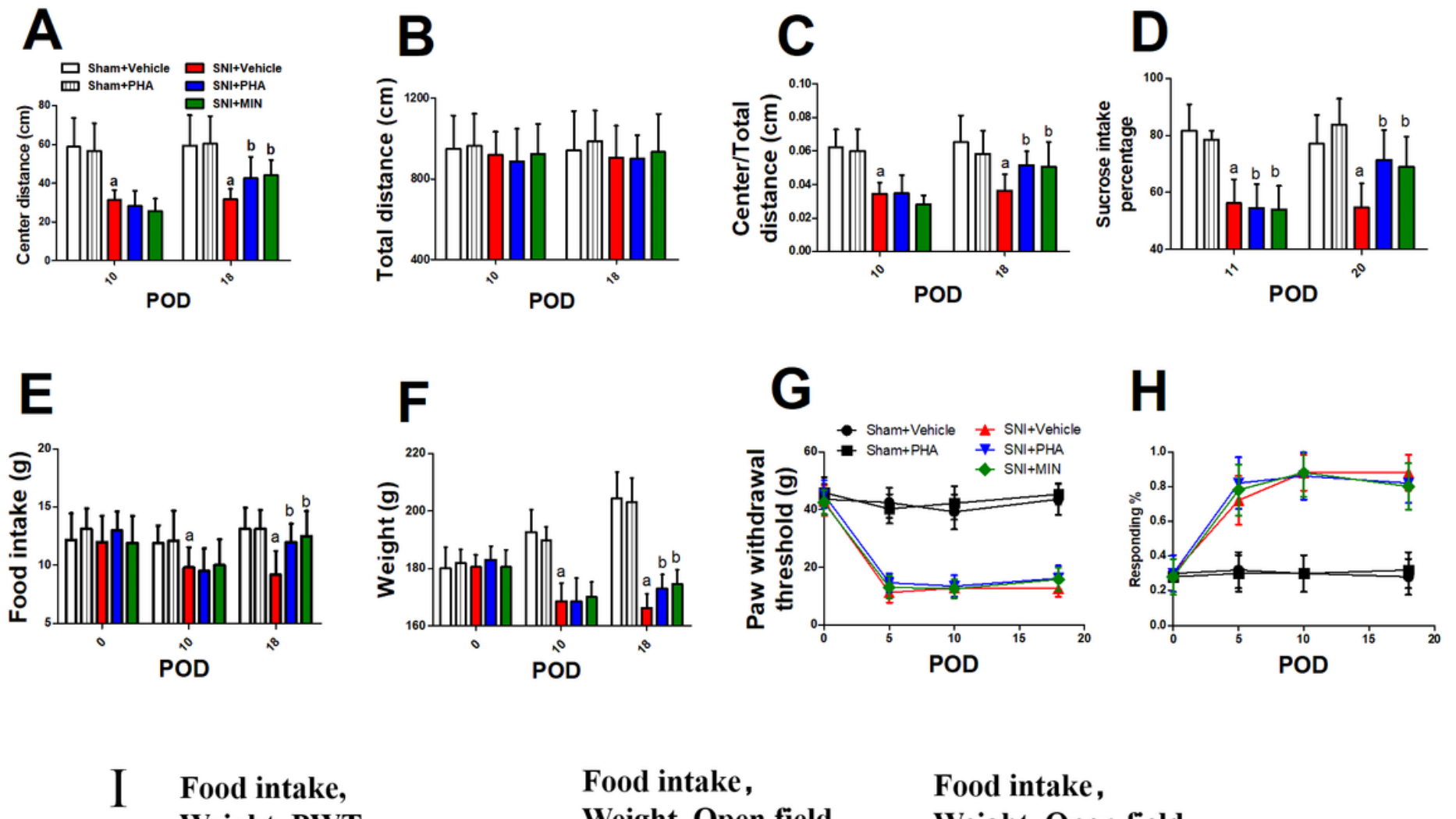

Food intake, Weight, PWT, Acetone

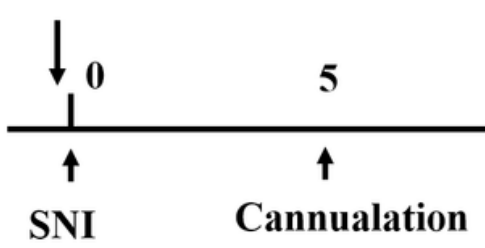

Food intake, Weight, Open field, PWT, Acetone,
Food intake, Weight, Open field, PWT, Acetone,

\section{Figure 2}

ICV injection of a7 nAch receptor agonist PHA and MIN decreased depression-like behaviors after SNI surgery. ICV PHA or MIN improved that decrement of center distance (A) and center/total distance (C) after SNI surgery on POD18. (B) SNI surgery did not affect total running distance in the open field test after surgery. (D) SNI decreased sucrose intake percentage from POD10 to POD18 and ICV PHA or MIN decreased that effect on POD18. SNI surgery decreased food intake (E) and weight gain (F) from POD10 to POD18 compared to sham group, and ICV PHA or MIN decreased that effect on POD18. ICV PHA or MIN did not affect paw withdrawal threshold $(\mathrm{G})$ and response to acetone $(\mathrm{H})$ after SNI surgery. (I) Experimental procedures and timeline. "a" denotes the statistically significant difference compared to the sham + vehicle group. "b" denotes the statistically significant difference compared to the SNI + vehicle group. a7 nAch alpha 7 nicotinic acetylcholine, PHA PHA-543613, ICV intracerebroventricular, MIN minocycline 

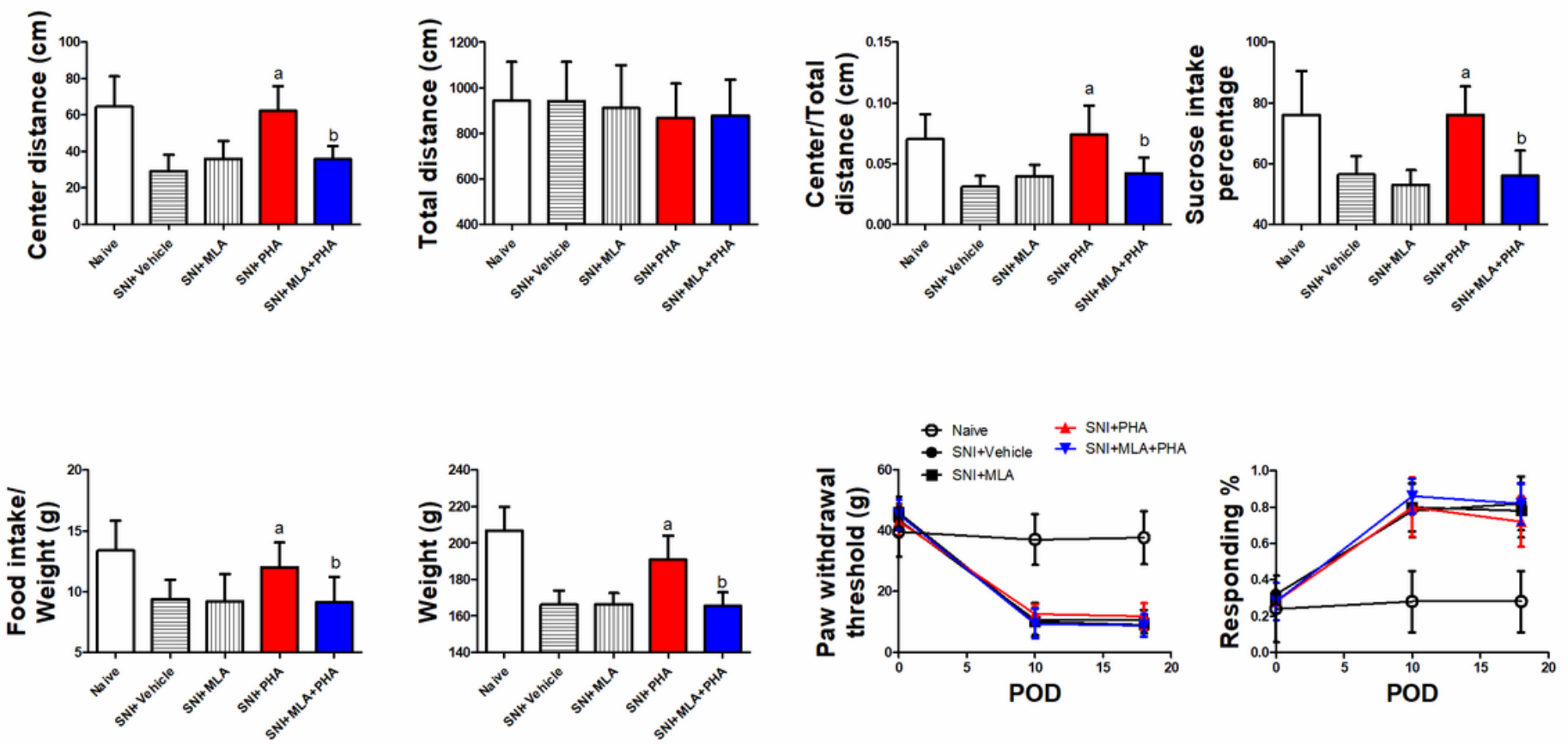

Figure 3

MLA decreased anti-depressive effect of PHA. ICV injection of a7 nAch receptor antagonist MLA decreased anti-depressive effect of PHA in center distance (A) and center/total distance (C) in the open field test and the total distance (B) running was not different between groups after SNI surgery on POD18. PHA increased sucrose intake percentage (D) and food intake (E) and weight gain (F) after SNI, while ICV MLA decreased that effect. MLA and PHA did not affect the paw withdrawal threshold (G) and response to acetone $(H)$. "a" denotes the statistically significant difference compared to the SNI + vehicle group. "b" denotes the statistically significant difference compared to the SNI + PHA group. MLA methyllycaconitine citrate 

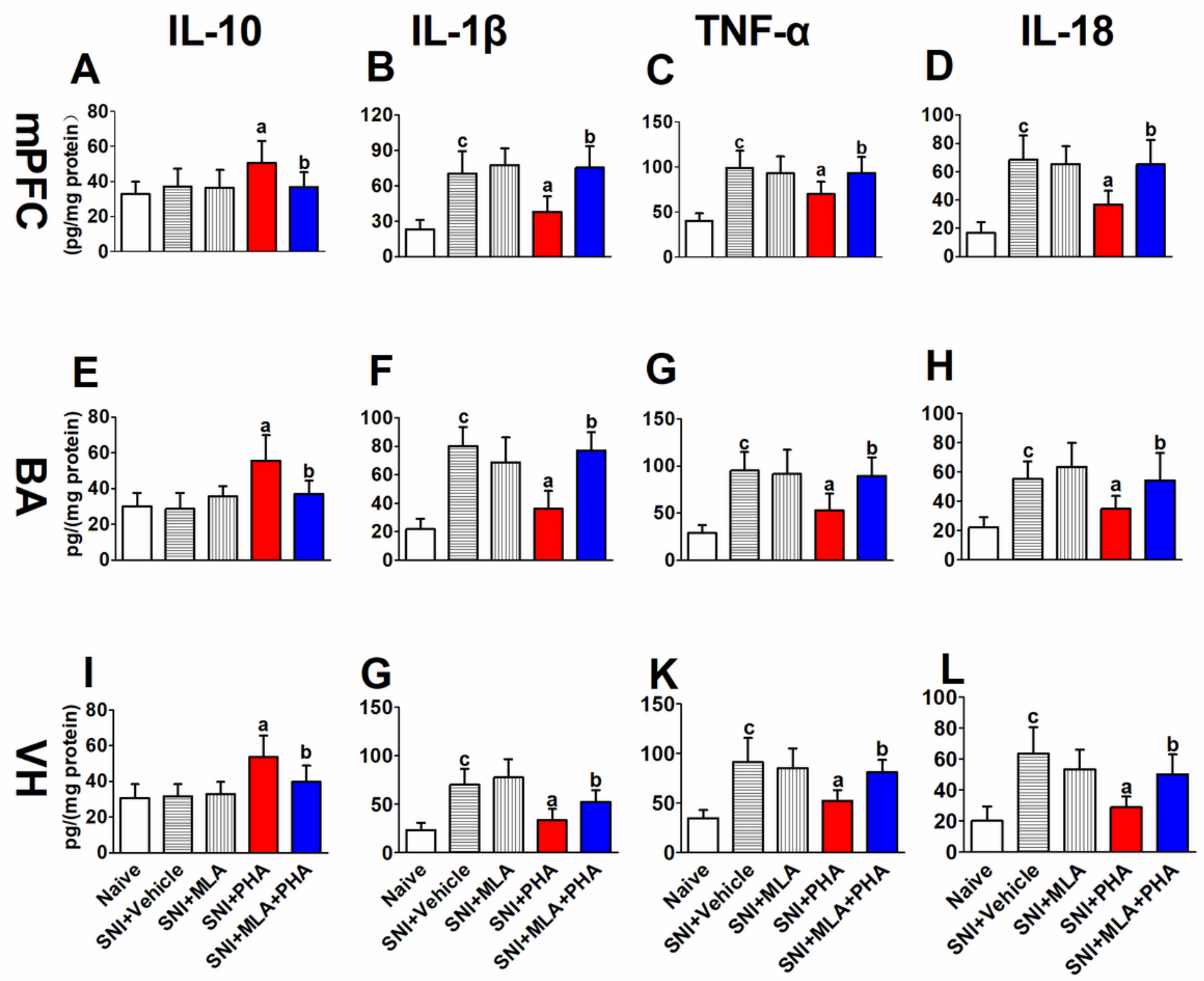

Figure 4

The effect of PHA on brain cytokines production. ICV PHA increased IL-10 in the MPFC (A), BA (E) and VH (I) compared to SNI+Vehicle group, and MLA decreased that effect. SNI increased IL-1 $\beta$ in the mPFC (B), BA (F) and VH (J) compared to Naive group, and ICV PHA decreased IL-1 $\beta$ in the mPFC, BA and VH compared to SNI+Vehicle group, while MLA decreased the effect of PHA. SNI increased TNF-a (C, G and $\mathrm{K})$ and IL-18 (D, H and L) in the mPFC, BA and VH compared to Naive group, and ICV PHA decreased their content in the MPFC, BA and VH compared to SNI+Vehicle group, while MLA decreased the effects of PHA. "a" denotes the statistically significant difference compared to the SNI + vehicle group. "b" denotes the statistically significant difference compared to the SNI + PHA group. ELISA enzymelinked immuneabsorbent assay. 

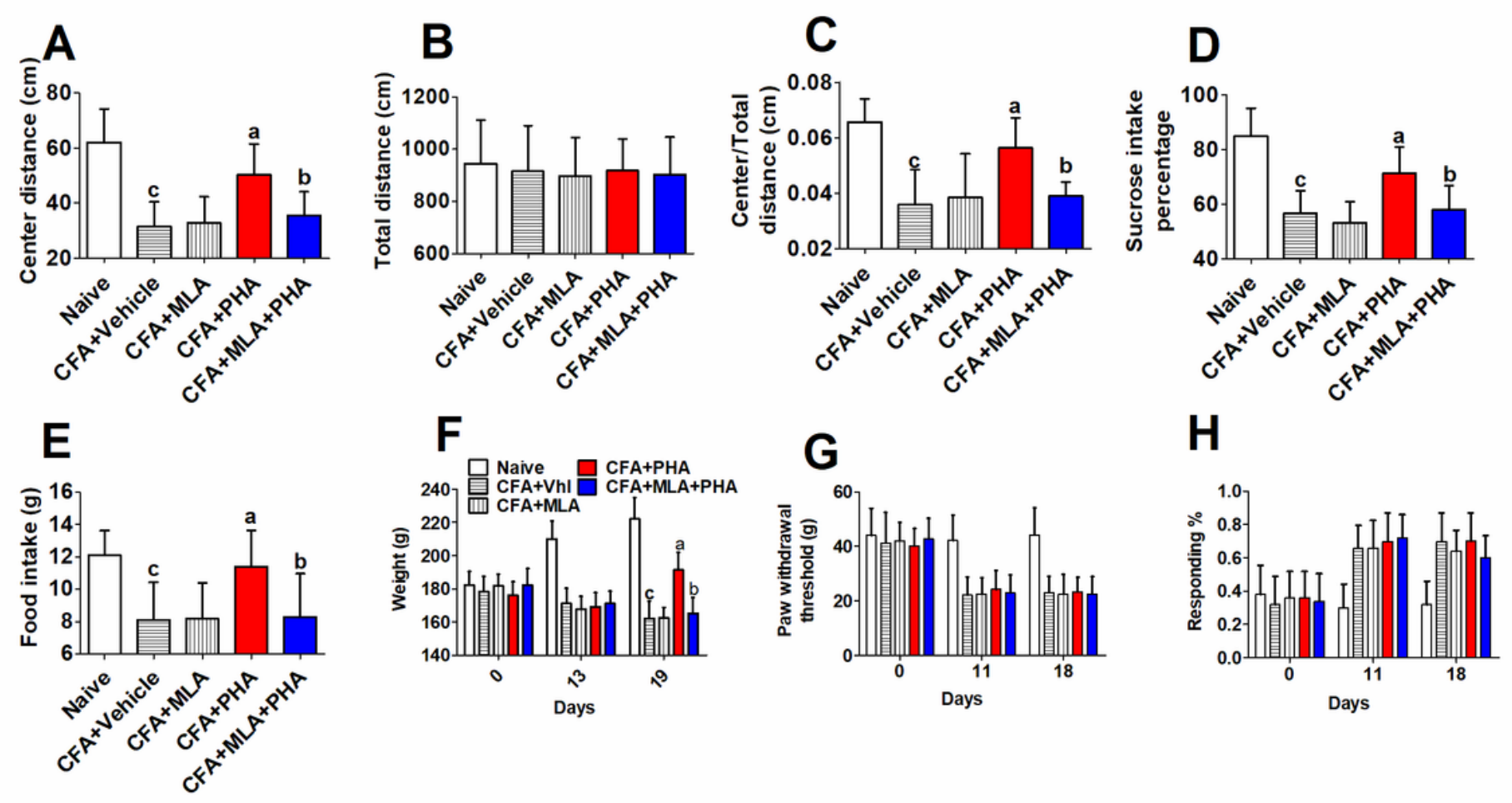

\section{Figure 5}

PHA decreased depressive-like behaviors induced by CFA. CFA decreased center distance (A) and center/total distance (C) in the open field test, and did affect total running distance moved (C), compared to naïve group, and PHA improved the behaviors in the open filed tests, whereas MLA decreased the effect of PHA. CFA decreased sucrose intake percentage (D), food intake (E) and weight gain (F), and PHA improved that effect, and the effects of PHA was abrogated by MLA. CFA decreased mechanical anodynia $(\mathrm{G})$ and increased response $(\mathrm{H})$ to cold, whereas ICV PHA and MLA did not affect that response. "a" denotes the statistically significant difference compared to the CFA + vehicle group. "b" denotes the statistically significant difference compared to the CFA + PHA group. 

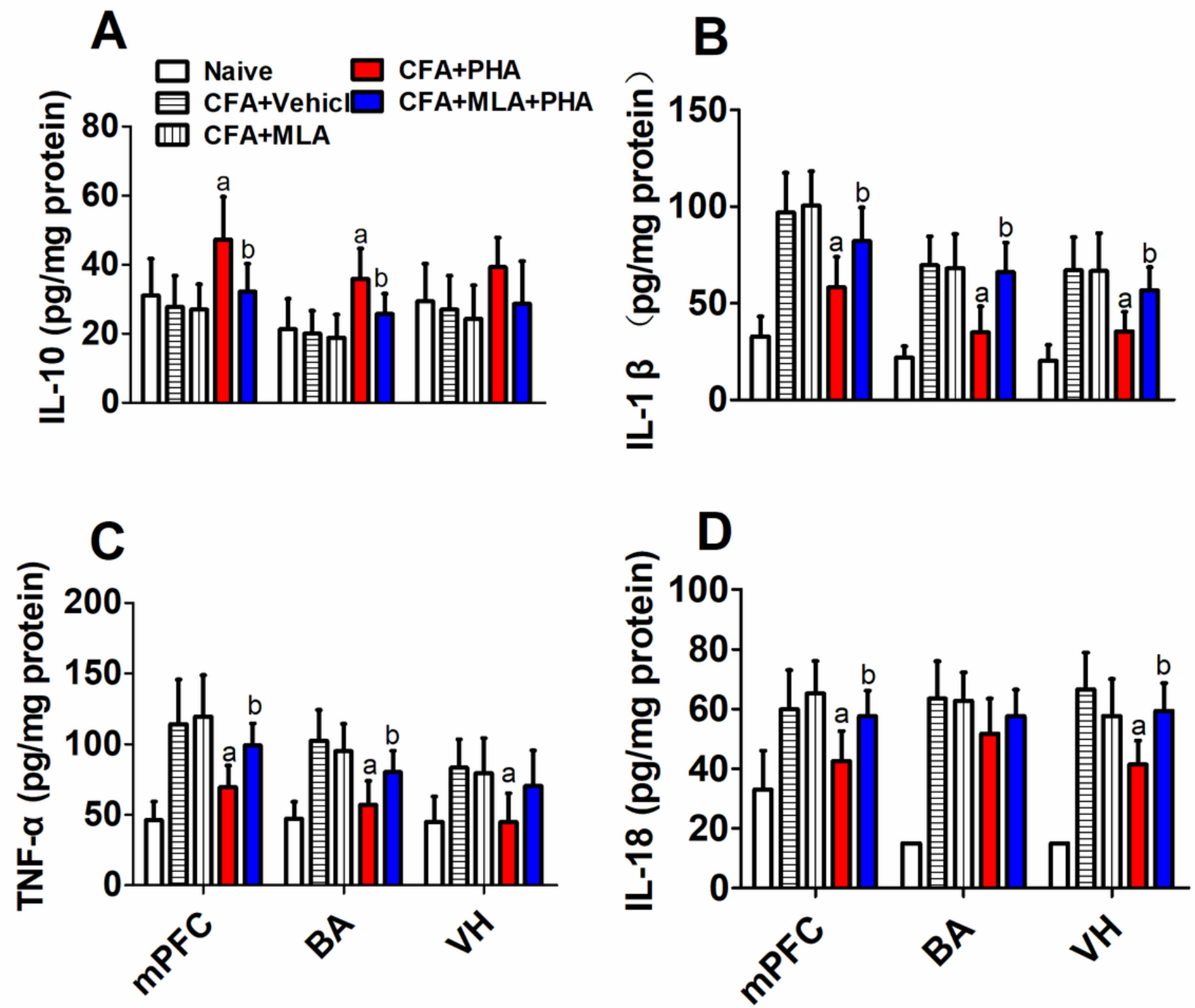

Figure 6

PHA decreased CFA-induced brain inflammation by activation of a7 nAch receptors. (A) ICV PHA increased IL-10 content in the MPFC and BA after CFA injection, and the effect of PHA was decreased by ICV MLA. (B) CFA increased IL-1 $\beta$ content in the MPFC BA and VH, and ICV PHA decreased IL-1 $\beta$ increment in that sites, and the effect of PHA was decreased by MLA after CFA injection. (C) CFA increased TNF-a content in the MPFC BA and VH, and ICV PHA decreased TNF- $a$ content in the MPFC, BA and $\mathrm{VH}$, the effect of PHA was abolished by MLA. (D) CFA increased IL-18 content in the MPFC BA and $\mathrm{VH}$, and ICV PHA decreased IL-18 content in the MPFC and VH, while the effect of PHA was decreased by MLA. "a" denotes the statistically significant difference compared to the CFA + vehicle group. "b" denotes the statistically significant difference compared to the CFA + PHA group. 

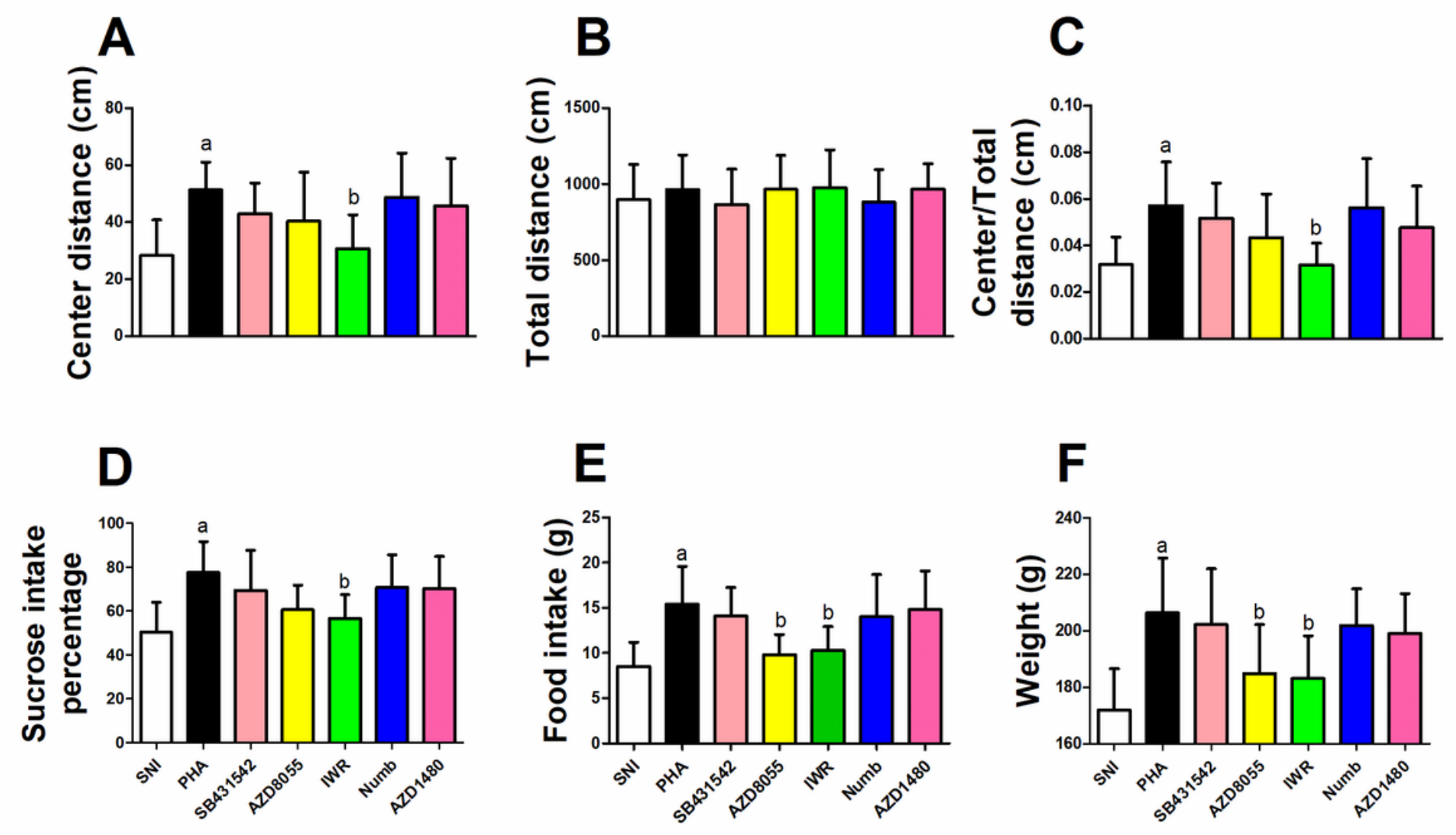

Figure 7

PHA decreased depressive-like behaviors via WNT/ $\beta$-catenin signal pathway. IWR (WNT/ $\beta$-catenin inhibitor) reversed the anti-depressive effect of PHA on center distance (A), center/total distance ratio (C), sucrose intake percentage $D$, food intake percentage $(E)$ and weight gain $(F)$, while did not affect total running distance(B) in the open field test after SNI surgery. However, AZD8055 (mTOR inhibitor) also reversed the effect of PHA on food intake percentage (E) and weight gain (F), Whereas SB431542(TGF $\beta$ inhibitor), Numb (notch inhibitor) and AZD1480 (JAK inhibitor) did not affect anti-depressive behaviors of PHA. "a" denotes the statistically significant difference compared to the SNI group. "b" denotes the statistically significant difference compared to the PHA group. 

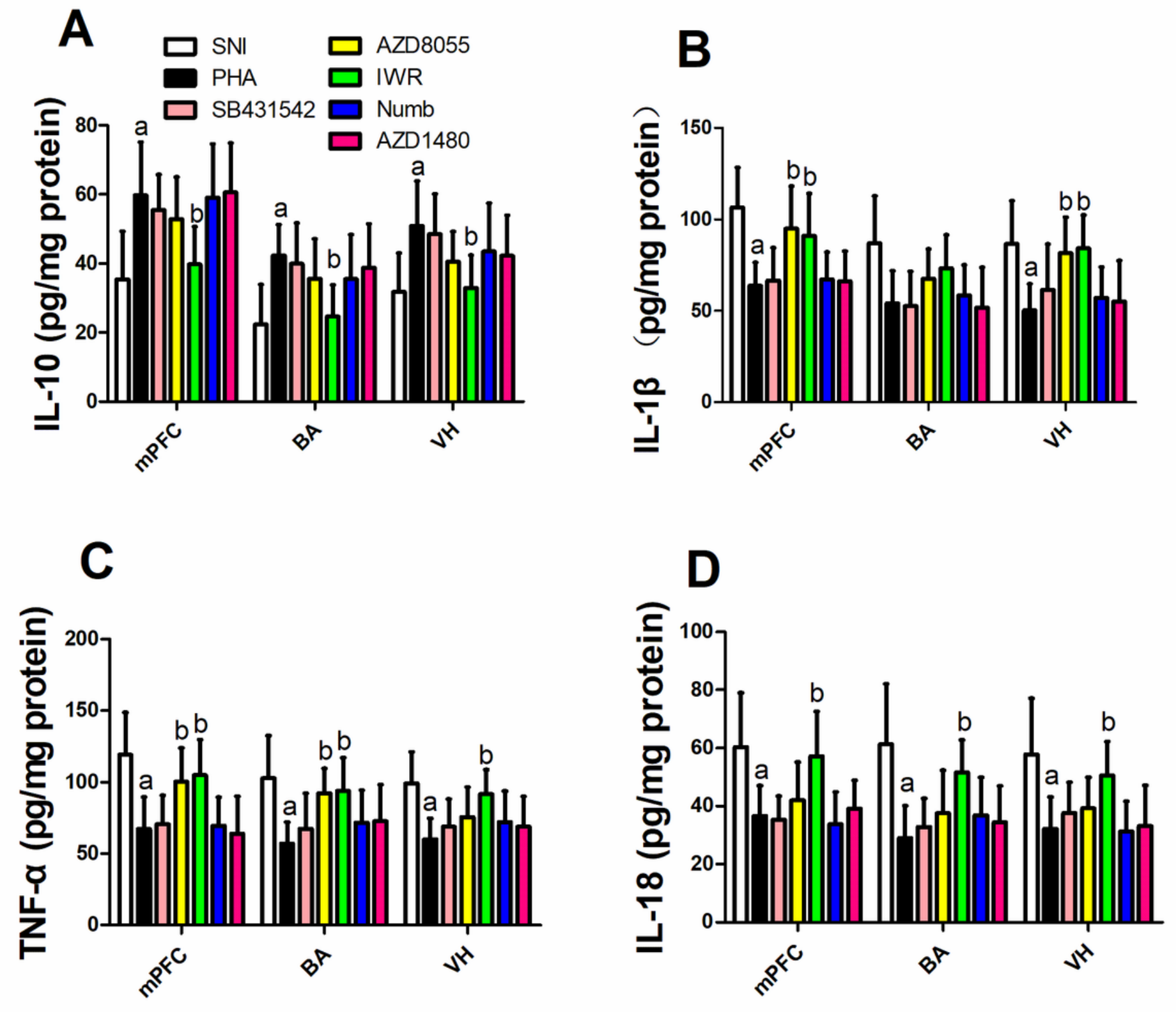

Figure 8

PHA decreased brain inflammation via WNT/ $\beta$-catenin and mTOR pathway. PHA increased IL-10 (A) and decreased IL-18 (D) in mPFC, BA and VH after SNI surgery, and IWR decreased those effect. (B) PHA decreased IL-1 $\beta$ content in mPFC and VH after SNI surgery, the effect was reversed by AZD8055 and IWR. (C) PHA decreased the content of TNF in MPFC, BA and VH after SNI surgery, and the content change of TNFa in MPFC and BA was reversed by AZD8055 and IWR. "a" denotes the statistically significant difference compared to the SNI group. "b" denotes the statistically significant difference compared to the PHA group. 